\title{
Observations of Breaking Waves and Energy Dissipation in Modulated Wave Groups
}

\author{
DAVID W. WANG AND Hemantha W. WiJESEKERA \\ Naval Research Laboratory, Stennis Space Center, Mississippi
}

(Manuscript received 31 October 2017, in final form 25 September 2018)

\begin{abstract}
It has been recognized that modulated wave groups trigger wave breaking and generate energy dissipation events on the ocean surface. Quantitative examination of wave-breaking events and associated turbulent kinetic energy (TKE) dissipation rates within a modulated wave group in the open ocean is not a trivial task. To address this challenging topic, a set of laboratory experiments was carried out in an outdoor facility, the Oil and Hazardous Material Simulated Environment Test Tank (203 m long, $20 \mathrm{~m}$ wide, $3.5 \mathrm{~m}$ deep). TKE dissipation rates at multiple depths were estimated directly while moving the sensor platform at a speed of about $0.53 \mathrm{~m} \mathrm{~s}^{-1}$ toward incoming wave groups generated by the wave maker. The largest TKE dissipation rates and significant whitecaps were found at or near the center of wave groups where steepening waves approached the geometric limit of waves. The TKE dissipation rate was $O\left(10^{-2}\right) \mathrm{W} \mathrm{kg}^{-1}$ during wave breaking, which is two to three orders of magnitude larger than before and after wave breaking. The enhanced TKE dissipation rate was limited to a layer of half the wave height in depth. Observations indicate that the impact of wave breaking was not significant at depths deeper than one wave height from the surface. The TKE dissipation rate of breaking waves within wave groups can be parameterized by local wave phase speed with a proportionality breaking strength coefficient dependent on local steepness. The characterization of energy dissipation in wave groups from local wave properties will enable a better determination of near-surface TKE dissipation of breaking waves.
\end{abstract}

\section{Introduction}

The breaking of dominant waves plays a key role in the exchange of momentum, mass, and heat in the air-sea interface (Soloviev and Lukas 2003; Babanin et al. 2010). The breaking process limits wave growth by removing wave energy through turbulent kinetic energy (TKE) dissipation. Several investigators report that wave breaking generates large turbulent vortices (Melville et al. 2002; Pizzo and Melville 2013), which significantly enhance mixing in the ocean boundary layer (Thomson et al. 2016) and gas exchange (Zappa et al. 2007). For example, Gemmrich and Farmer (2004) showed TKE dissipation rate per unit mass $\varepsilon$ at $1-\mathrm{m}$ depth prior to the wave breaking was about $5 \times$ $10^{-6} \mathrm{~W} \mathrm{~kg}^{-1}$ and increased to about $8 \times 10^{-3} \mathrm{~W} \mathrm{~kg}^{-1}$ during the wave breakings. The enhanced dissipation occurs within a layer less than one wave height and decays with depth following a power law, $\varepsilon \sim Z^{-m}$, where $Z$ is the distance from the mean water surface and $m$

Corresponding author: David W. Wang, david.wang@nrlssc. navy.mil is a coefficient that varies between 1 and 3 (Sutherland and Melville 2015; Thomson et al. 2016). More recently, Gemmrich (2010), Thomson et al. (2016), and Lee et al. (2017) measured $\varepsilon$ from a wave-following reference frame and found similar power-law dependence. However, high dissipation rates for the wave following measurement are limited to a depth of about $10 \%$ of wave height.

Duncan $(1981,1983)$ scaled the breaking wave dissipation using wave properties, and showed that TKE dissipation rate per unit length of breaking wave crest $\varepsilon_{L}$ can be expressed as $\varepsilon_{L}=b \rho C_{b}^{5} / g$, where $b$ is the breaking strength coefficient, $g$ is the gravitational acceleration, and $C_{b}$ is the phase speed of breaking waves. Drazen et al. (2008) show a breaking wave strength dependence on wave steepness based on an inertial scaling model for plunging breakers. Romero et al. (2012) show a similar steepness dependence using existing laboratory data. Field data studies show that $b$ can be parameterized by the wave age or the significant wave steepness estimated from wave spectral properties (e.g., Zappa et al. 2016).

Theoretical and laboratory studies show that wave breaking is initiated through the Benjamin-Feir (BF) 

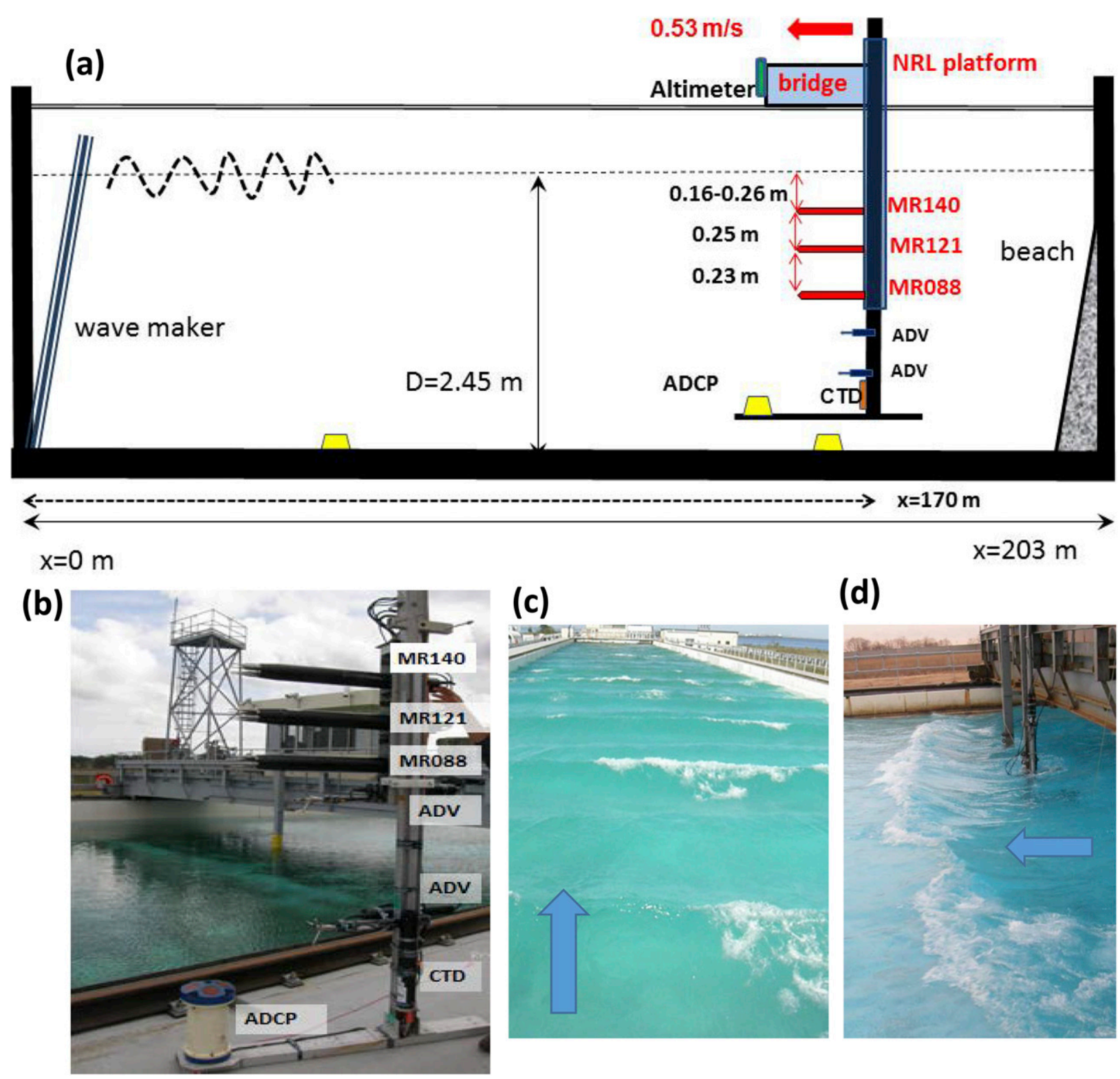

\section{(c)}

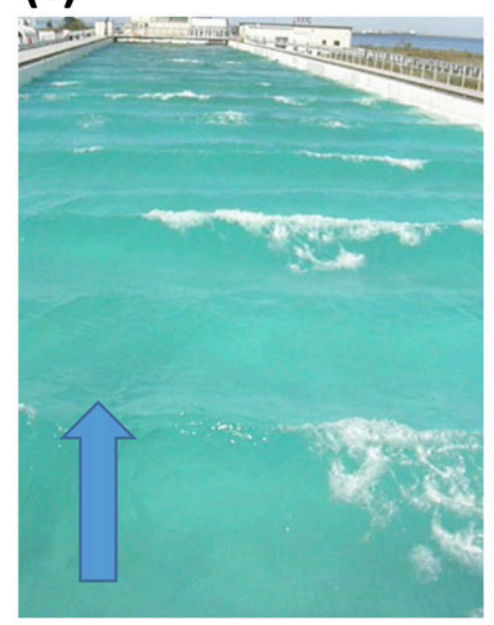

(d)

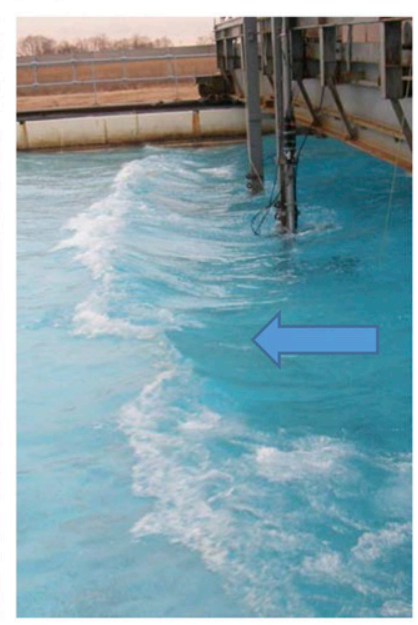

FIG. 1. (a) Schematic diagram of the wave tank and the NRL instrument platform and locations of three MRs (red), (b) photo of the NRL platform and mounted sensors, (c) periodic occurrence of whitecaps in the along-tank direction, and (d) whitecap of an individual wave breaking near the NRL platform. The arrows in (c) and (d) indicate wave propagation directions.

modulation instability mechanism (Benjamin and Feir 1967), in which a steepening wave breaks as it reaches the critical threshold of the local geometric, kinematic, and dynamic limits (Banner and Peirson 2007; Babanin et al. 2010; Perlin et al. 2013; Schwendeman and Thomson 2017). The onset of wave breaking is periodic and is closely related to wave group structures (Donelan et al. 1972; Holthuijsen and Herbers 1986; Banner et al. 2000). However, field studies of wave breaking and dissipation of energy within modulated wave groups are rare owing to the challenges of identifying wave groups and making measurements in breaking waves. Therefore, wave-breaking studies are often conducted through laboratory experiments, where wave group modulation and breaking can be studied under wellcontrolled environments (Melville 1982,1983; Rapp and Melville 1990; Banner and Tian 1998; Tulin and Waseda 1999; Banner and Peirson 2007; Babanin et al. 2007, 2010). During laboratory experiments the breaking wave energy dissipation has been estimated by measuring potential wave energy before and after breaking (Perlin et al. 2013). The TKE dissipation of breaking 
TABLE 1. Summary of initial monochromatic wave conditions and MR sensor depths for the nine test runs. The $T_{i}$ and $H_{i}$ are initial monochromatic wave period and height, respectively. The $z$ is sensor depth below tank water line. The initial wave steepness is calculated as $a k$, where $a=H_{i} / 2$ and $k=(2 \pi)^{2} / g T_{i}^{2}$ and $g$ is the gravity acceleration. The $\Delta T$ is the time duration of selected data segments for each test run.

\begin{tabular}{|c|c|c|c|c|c|c|c|}
\hline \multirow[b]{2}{*}{ Test No. } & \multicolumn{2}{|c|}{ Wave } & \multirow{2}{*}{$\frac{\text { Steepness }}{a k}$} & \multirow{2}{*}{$\frac{\mathrm{MR} 140}{z(\mathrm{~m})}$} & \multirow{2}{*}{$\frac{\mathrm{MR} 121}{z(\mathrm{~m})}$} & \multirow{2}{*}{$\frac{\text { MR088 }}{z(\mathrm{~m})}$} & \multirow[b]{2}{*}{$\Delta T(\mathrm{~s})$} \\
\hline & $T_{i}(\mathrm{~s})$ & $H_{i}(\mathrm{~m})$ & & & & & \\
\hline 1 & 1.5 & 0.27 & 0.24 & 0.16 & 0.41 & 0.64 & 80 \\
\hline 2 & 1.5 & 0.27 & 0.24 & 0.16 & 0.41 & 0.64 & 80 \\
\hline 3 & 1.5 & 0.27 & 0.24 & 0.19 & 0.44 & 0.67 & 80 \\
\hline 4 & 1.5 & 0.27 & 0.24 & 0.21 & 0.46 & 0.69 & 70 \\
\hline 5 & 1.5 & 0.27 & 0.24 & 0.26 & 0.51 & 0.74 & 90 \\
\hline 6 & 1.71 & 0.4 & 0.28 & 0.16 & 0.41 & 0.64 & 60 \\
\hline 7 & 1.71 & 0.4 & 0.28 & 0.19 & 0.44 & 0.67 & 80 \\
\hline 8 & 1.71 & 0.4 & 0.28 & 0.21 & 0.46 & 0.69 & 80 \\
\hline 9 & 1.71 & 0.4 & 0.28 & 0.26 & 0.51 & 0.74 & 110 \\
\hline
\end{tabular}

waves in modulated wave groups and the associated vertical structure are poorly known mainly because of difficulties of making reliable and direct measurements of dissipation rates within the wave groups (Lee et al. 2017).

The goal of this study is to quantify the variability of TKE dissipation rates and associated vertical distributions within wave groups. Here we discuss the analysis of turbulent velocity measurements collected from three turbulent sensing packages mounted on the towed platform of the Oil and Hazardous Material Simulated Environment Test Tank (Ohmsett) facility in Leonardo, New Jersey. As discussed below, turbulent velocity shears were measured to estimate TKE dissipation rates as typically used in analyses of oceanic observations (Lueck et al. 2002). Section 2 provides a description of the experiment facility and instrumentation setups. Section 3 discusses measurement procedure and data processing. Results and discussion are given in sections 4 and 5, respectively. Finally, conclusions are presented in section 6 .

\section{Experiment facility and instrumentation}

\section{a. Wave tank and instrument platform}

The outdoor Ohmsett facility is $203 \mathrm{~m}$ long, $20 \mathrm{~m}$ wide, and $3.5 \mathrm{~m}$ deep (Fig. 1a). The tank is filled with saltwater with salinity 34.5 psu to a depth of $2.45 \mathrm{~m}$ during the experiment and is equipped with a bottomhinged flap-type wave maker with an adjustable-paddle stroke frequency and distance (Asher 2005). The wave maker is at the south end of the tank, and a wavedamping beach system is at the north end to reduce wave reflection of incoming waves. During the experiment, monochromatic waves with initial wave steepness of about 0.24 and 0.28 were generated for two paddle-stroke periods of 1.5 and $1.71 \mathrm{~s}$, respectively
(Table 1). These steep monochromatic waves are similar to those used in the experiment by Babanin et al. (2010).

The microstructure measurements were made from sensors mounted on an instrumentation platform (also referred to as the NRL platform; Figs. 1a,b). The instrument platform was constructed by combining a cylindrical pole (hereafter "pole") attached to the 2.3-m-long $\mathrm{H}$ beam. The NRL platform was connected to the movable main tank bridge to make underway measurements. In this setup, only the $\mathrm{H}$ beam was attached rigidly to the bridge (Figs. 1a,b). The pole was mounted on the beam in such a way that the pole could be moved vertically (up and down) along the beam to position the microstructure sensors [MicroRider-1000 (MR)] at different depths. Three microstructure sensing packages (MR140, MR121, and MR088) and an acoustic Doppler velocimeter (ADV) were mounted on the pole. Another ADV and a conductivity-temperature-depth (CTD) package were mounted on the $\mathrm{H}$ beam at fixed depths (Figs. 1a,b). An upward-looking acoustic Doppler current profiler (ADCP) was mounted on a horizontal extension attached to the bottom of the H beam (Fig. 1b). Note that all of the sensing elements were directed toward the wave maker. The flow field around the instrument platform can be distorted as waves pass the moving platform. Therefore, all three MRs were mounted on the pole, so that the turbulent sensors were placed about $0.8 \mathrm{~m}$ from the center of the $\mathrm{H}$ beam to prevent sensors from being exposed to a distorted flow field (Fig. 1a). Note that even if reflected flows from the moving beam would have a speed similar to the wave orbital velocity, the position of the sensor tip is at least $0.6 \mathrm{~m}$ ahead of any reflected flow. More details about the experiment setup and facilities can be found at Wang and Wijesekera (2017). 


\section{b. Microstructure package (MR)}

Turbulent velocity fluctuations were measured by shear probes in the MR. The MR is $1 \mathrm{~m}$ long and $\sim 10 \mathrm{~cm}$ in diameter, weighs $5.5 \mathrm{~kg}$ in air, and is manufactured by Rockland Scientific Inc. (http://rocklandscientific.com/ products/modular-systems/microrider/). The nose cone of the MR holds two shear probes, two fast-response thermistors, and high-resolution pressure, acceleration, and tilt sensors. The instrument is powered by $9-18$ volts $\mathrm{dc}$, and data are recorded internally. The sampling rate of shear probes and fast thermistors is $512 \mathrm{~Hz}$, and the other sensors sample at $64 \mathrm{~Hz}$.

\section{Measurements}

\section{a. Measurement procedure}

During the experiment, the bridge and instrument platform were first positioned at a distance of $170 \mathrm{~m}$ from the wave maker before starting the wave maker (Fig. 1a). Once monochromatic waves were generated, the bridge-instrument platform moved at a steady towing speed of $0.53 \mathrm{~m} \mathrm{~s}^{-1}$ toward the wave maker from its initial position to a location $35 \mathrm{~m}$ from the wave maker, while encountering wave-breaking events and collecting concurrent measurements of microscale velocity shear, currents, and temperature and salinity in the tank. To avoid the impact from waves reflected back from the beach end on the measurements, we conducted measurements before the reflected waves reached the instrument platform. The waves with periods 1.5 and $1.71 \mathrm{~s}$ reached the instrument platform within 240 and 290 s, respectively, after the start of wave actions. During the experiment, microscale velocity shears were measured at three depths with vertical spacing of about 0.23 and $0.25 \mathrm{~m}$ (Fig. 1b). We repeated the measurement procedure by adjusting the vertical position of the pole at increments of $2-5 \mathrm{~cm}$ to construct a vertical profile of TKE dissipation rate from 0.16 to $0.74 \mathrm{~m}$ below the water level.

\section{b. TKE dissipation rate}

For homogeneous isotropic turbulence, the TKE dissipation rate per unit mass $\varepsilon\left(\mathrm{W} \mathrm{kg}^{-1}\right)$ is computed from the variance of the turbulent velocity gradients (e.g., Tennekes and Lumley 1972):

$$
\varepsilon=7.5 \nu\left\langle u_{x}^{2}\right\rangle,
$$

where $\left\langle u_{x}^{2}\right\rangle$ is the variance of the velocity shear $u_{x}\left(\mathrm{~s}^{-1}\right)$, $\nu$ is the kinematic molecular viscosity $\left(\mathrm{m}^{2} \mathrm{~s}^{-1}\right)$, and the angle brackets denote either time or space average. In practice, the velocity-shear variance $\left\langle u_{x}^{2}\right\rangle$ is estimated directly by integrating the shear spectrum for a specified wavenumber band ranging from a low wavenumber representing inertial subrange motions to the dissipation wavenumber [or the Kolmogorov wavenumber $\left(\varepsilon / \nu^{3}\right)^{1 / 4}$ ]. However, when turbulent motions are highly energetic, such as during surface-wave-breaking events, the shear probes used in microstructure sensing packages do not fully resolve the shear spectrum at higher wavenumbers, thus underestimating the TKE dissipation rate. Therefore, the area under the measured velocity-shear spectra represents only a fraction of the total variance of shear. The Nasmyth universal spectrum or the theoretical spectral shape in the inertial subrange can be related to the TKE dissipation rate as (Oakey 1982)

$$
\phi(k)=A_{1} \alpha(2 \pi)^{4 / 3} \varepsilon^{2 / 3} k^{1 / 3},
$$

where $A_{1}=24 / 55$ for the transverse spectrum and $\alpha=$ 1.5 is the universal constant. Note that the wavenumber $k$ is in cycles per meter (cpm). The dissipation rate can be estimated by fitting the theoretical form of $\phi(k)$ in (2) to the observed shear spectrum $\phi_{M}(k)$ in the inertial subrange wavenumber band from $k_{a}$ to $k_{b}$. By combining (2) and the observed transverse wavenumber spectrum $\phi_{M}(k)$, we determined the TKE dissipation rate as (e.g.,Tennekes and Lumley 1972; Bluteau et al. 2016)

$$
\varepsilon=\left[\frac{\frac{4}{3} E}{A_{1} \alpha(2 \pi)^{4 / 3}\left(k_{b}^{4 / 3}-k_{a}^{4 / 3}\right)}\right]^{3 / 2},
$$

where $E=\int_{k_{a}}^{k_{b}} \phi_{M}(k) d k$.

During the experiments, the two shear probes in each MR were mounted orthogonally to each other to measure the cross-stream and vertical velocity gradients while the sensor platform was moving along the tank or the along-stream direction. We computed transverse spectra of velocity shear for the two orthogonal components (cross-stream and vertical) and then averaged both together to construct a representative spectrum for a selected data segment $\phi_{M}(k)$, where $k$ is the wavenumber in the direction of the mean flow (i.e., along the tank axis). The measurements from the moving platform enable the conversion of the velocity-shear frequency spectrum into a wavenumber spectrum using Taylor's frozen turbulence hypothesis. For a sampling rate of $512 \mathrm{~Hz}$ and a platform speed of $0.53 \mathrm{~m} \mathrm{~s}^{-1}$, velocity fluctuations can be resolved at 1-mm spatial scales. Velocity shear frequency spectra $\phi_{M}(f)$ were calculated for 1-s segments of velocity-shear measurements. The frequency spectra $\phi_{M}(f)$ were converted to the wavenumber spectrum, where $\phi_{M}(k)=U_{B} \phi_{M}(f)$, $k=f / U_{B}, U_{B}$ is the towing speed of the bridge $\left(\mathrm{m} \mathrm{s}^{-1}\right)$, 
and $f$ is the frequency $(\mathrm{Hz})$. Examples of velocity shear spectra for 1-s data segments during a test run for three different dissipation levels are illustrated in Fig. 2. The TKE dissipation rate [(3)] was evaluated first by averaging spectral estimates from two shear probes and then by integrating the averaged shear spectrum between $k_{a}=10 \mathrm{cpm}$ and $k_{b}=35 \mathrm{cpm}$. Estimated TKE dissipation rates based on (3) for the spectra shown in Fig. 2 are $1.26 \times 10^{-5}, 1.72 \times 10^{-4}$, and $3.14 \times 10^{-3} \mathrm{~W} \mathrm{~kg}^{-1}$, respectively. To illustrate overall velocity shear spectrum including inertial and dissipation ranges, we reconstructed Nasmyth spectra for the three estimated dissipation rates (Oakey 1982).

TKE dissipation rates were computed from 1-s segments of velocity shear data. The 1-s dissipation estimates were adequate for the examination of dissipation rates within wave groups of $\sim 5-10 \mathrm{~s}$. However, the 1 -s estimates were not sufficient to examine the variability of TKE dissipation rate in individual waves generated by the wave maker. Therefore, a bandpass-filtered velocity shear squared $s h^{2}$ was used as an indicator to examine dissipation rate variability within individual waves, where $s h^{2}=\left(u_{x}^{f}\right)^{2}$ and $u_{x}^{f}$ is the band-passed turbulent velocity shear, filtered between 5.3 and $18.6 \mathrm{~Hz}$. Note that the corresponding wavenumbers of the band-passed frequencies of 5.3 and $18.6 \mathrm{~Hz}$ are 10 and $35 \mathrm{cpm}$, respectively, for a platform speed of $0.53 \mathrm{~m} \mathrm{~s}^{-1}$.

\section{c. Wave parameters}

During the experiment, surface elevations of monochromatic waves were obtained by measurements from pressure sensors in the MRs at a sampling rate of $512 \mathrm{~Hz}$ and by the downward-looking sonic altimeters mounted on the bridge platform that sampled at $10 \mathrm{~Hz}$. Surface wave elevations estimated from the altimeters and the pressure sensors were very similar. For a better synchronization with velocity shear measurements from the MRs, we used wave elevations derived from the pressure sensor in the MR121 (Fig. 1c). The local wave parameters, wave amplitude $a$, and peak frequency $f_{p}$ were derived from the variance and peak frequency of the wavelet energy spectrum (Huang and Hwung 2007), respectively. The local peak frequency was corrected for the Doppler shift due to the moving platform, and then the local steepness $\delta$ (Figs. 3d and 4 d) was estimated from $\delta=a \omega^{2} / g$, where $\omega=2 \pi f_{p}, a$ is the local wave amplitude, and $g$ is the gravitational acceleration. The estimated steepness is very similar to those defined in Rapp and Melville (1990) and Tian et al. (2010). Furthermore, the wave-group phase $\theta_{g}$ was derived (Figs. 3c and 4c) by applying the Hilbert transform to the envelope of wave groups represented

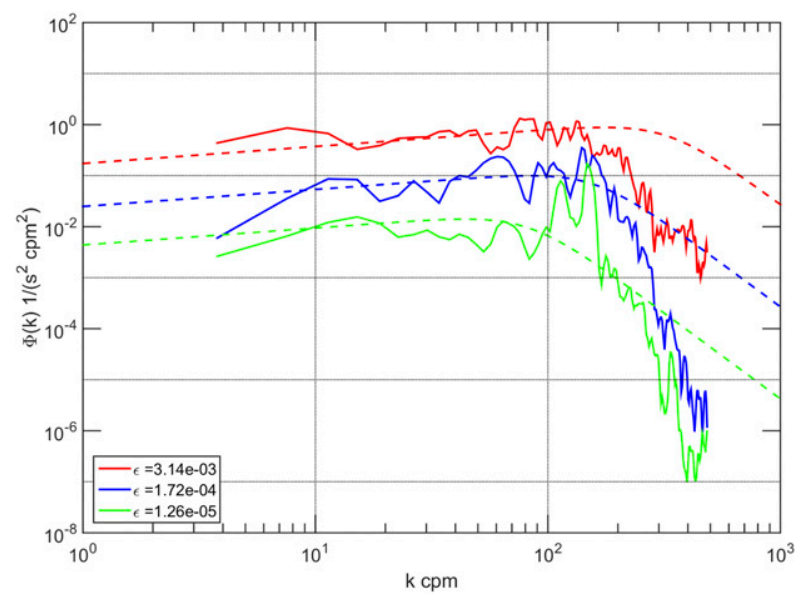

FIG. 2. Velocity shear wavenumber spectra for TKE dissipation rates of $1.26 \times 10^{-5}, 1.72 \times 10^{-4}$, and $3.14 \times 10^{-3} \mathrm{~W} \mathrm{~kg}^{-1}$, where dissipation rates were estimated from (3). The dashed curves represent the reconstructed Nasmyth spectra for the estimated dissipation rates. The velocity shear data are from MR140 at $0.19 \mathrm{~m}$ below water surface.

by the local wave amplitude (e.g., Melville 1982; Huang and Hwung 2007).

\section{Results}

\section{a. TKE dissipation rate within modulated wave groups}

TKE dissipation measurements were carried out under wave groups with frequent occurrences of wave breaking. Figure 1c shows a snapshot of whitecap formations from wave breaking during the experiment. The occurrences of whitecaps were evenly distributed in the along-tank direction with noticeable spatial patchiness. Figure 1d shows the NRL platform during such an event.

Here we analyzed microstructure turbulence and wave datasets from nine test runs for various wave conditions and MR depths (Table 1). Examples of two 45-s data segments representing initial monochromatic wave periods of 1.71 and $1.5 \mathrm{~s}$ are shown in Figs. 3 and 4, respectively. Modulated wave elevation shows welldefined group structures as depicted by envelopes of local wave amplitude (Fig. 3a) and time-frequency contours of wavelet spectra (Fig. 3b). The modulated wave group contains from four to seven individual waves and has a period of about $5-10 \mathrm{~s}$. The wave group phase angle $\theta_{g}$ is shown in Fig. 3c. Modulation instability increased wave steepness to the geometric limits at or near the center of the wave group and eventually led to a breaking onset while injecting turbulence into the water column. As a result, both $\delta$ and turbulent shear variance $s h^{2}$ peak near or at the center of the wave group. 


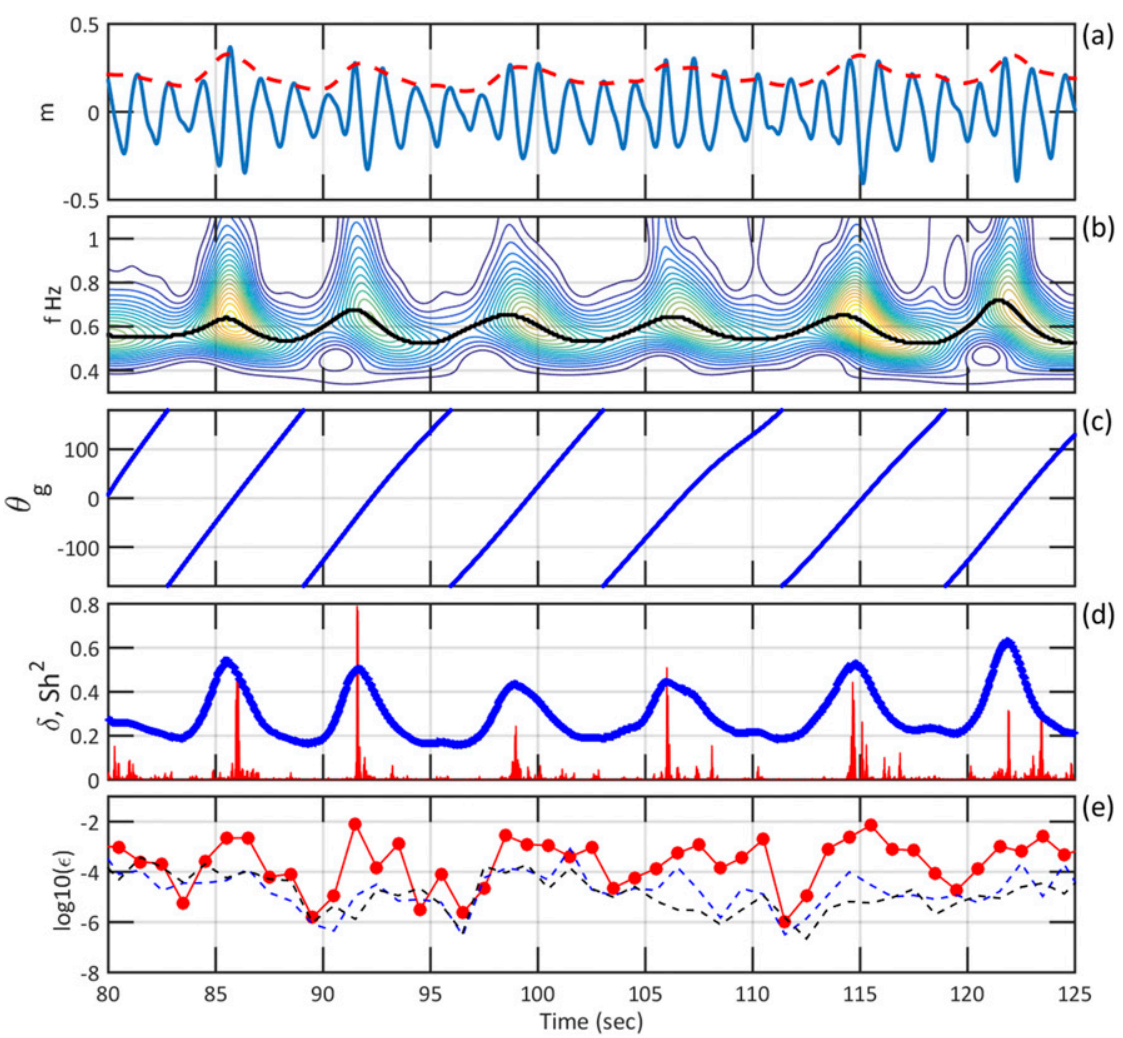

FIG. 3. A 45-s data segment of TKE dissipation measurements under wave groups generated by initial monochromatic waves with a period of $1.71 \mathrm{~s}$ : (a) surface wave elevation (blue) and envelope of modulated wave group (red dashed line), (b) wavelet spectrum and local peak wave frequency (black), (c) wave group phase $\theta_{g}$, (d) velocity shear squared $s h^{2}\left(\mathrm{~s}^{-2}\right)$ divided by a factor of 1000 (red) and wave steepness $\delta$ (blue), and (e) TKE dissipation rate $\left(\mathrm{W} \mathrm{kg}^{-1}\right)$ of 1 -s velocity shear segments from the MRs at depths of $z=0.19$ (red), 0.44 (blue), and $0.67 \mathrm{~m}$ (black).

The time series of $s h^{2}$ and $\varepsilon$ from (3) at $0.19,0.44$, and $0.67 \mathrm{~m}$ below the water level show modulation of $s h^{2}$ and $\varepsilon$ (Figs. $3 \mathrm{~d}, \mathrm{e}$ ) at the wave group period. The periodicity of TKE dissipation rate was more noticeable at the shallowest depth. Near-surface TKE dissipation at $0.19 \mathrm{~m}$ below the water surface varied in a periodic manner between $10^{-6}$ and $10^{-2} \mathrm{~W} \mathrm{~kg}^{-1}$, and the stronger dissipations were found during breaking events. The enhancement of near-surface TKE dissipation rate was highly correlated with the increasing wave steepness (Fig. 3d). The TKE dissipation rates at a depth of approximately one wave height below the water surface varied between $O\left(10^{-6}\right)$ and $O\left(10^{-4}\right) \mathrm{W} \mathrm{kg}^{-1}$ and were less correlated with the modulated wave steepness and the wave amplitude (Fig. 3d). Similar results were also observed in TKE measurements for monochromatic waves with a peak period of $1.5 \mathrm{~s}$ (Fig. 4).

Our observations indicate that local wave properties and TKE dissipation rates were highly modulated within the wave group of monochromatic waves with initial wave steepness of 0.24 and 0.28 (Table 1 ). By combining measurements from the nine test runs under the two initial monochromatic wave conditions, the dependence on the wave group is examined by expressing local wave amplitude, frequency, steepness $\delta$, and TKE dissipation rate as a function of wave group phase $\theta_{g}$ (Fig. 5). Here, the local wave properties are the averaged values of the 1-s data segment corresponding to velocity-shear segment for TKE dissipation rate estimate. Both wave amplitude and frequency were modulated and become largest near or at the center of the wave group (Figs. 5a,b). The modulation increases wave amplitude by $40 \%$ and wave frequency by $10 \%$, as group phase bin-averaged $\left\langle a \mid a_{m}\right\rangle$ and $\left\langle f_{p} \mid f_{p m}\right\rangle$ reach 1.4 and 1.1 , respectively. Here, $a_{m}$ and $f_{p m}$ are, mean values of local amplitude and frequency, respectively, for each selected data segment (Table 1). The increase of frequency implies a decrease of phase speed of a steepening wave, which is consistent with observations reported by Banner et al. (2014). 

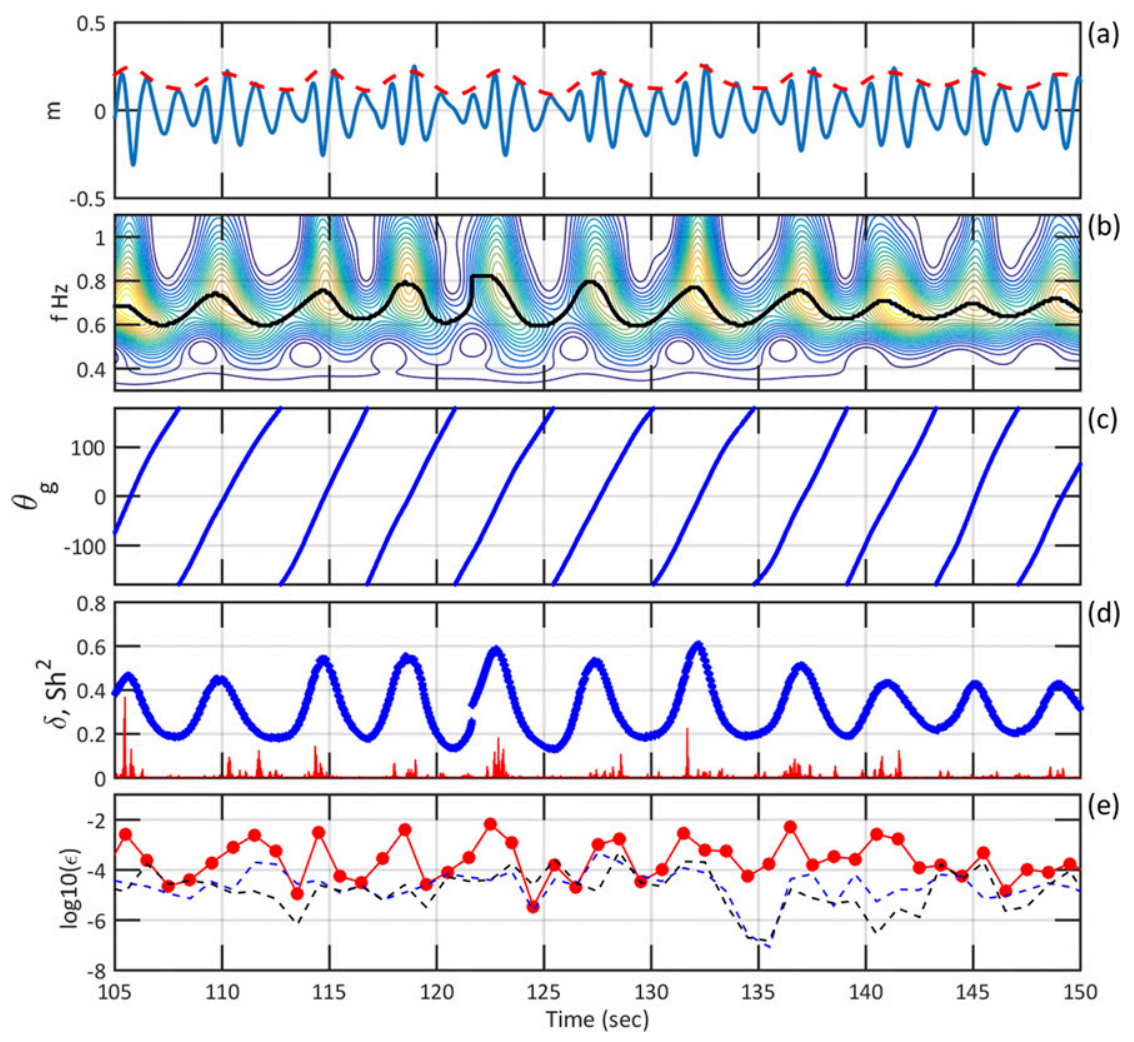

FIG. 4. As in Fig. 3, but for a period of $1.5 \mathrm{~s}$.

As a result of modulation in wave amplitude and frequency, both wave steepness and dissipation rate were largest for $\left|\theta_{g}\right|<50^{\circ}$, where wave group phase binaveraged steepness $\langle\delta\rangle$ and dissipation rate $\langle\varepsilon\rangle$ were about 0.35 and $3.5 \times 10^{-3} \mathrm{~W} \mathrm{~kg}^{-1}$, respectively. Away from the center of the wave group (i.e., prior to and after the active wave breaking), both $\langle\delta\rangle$ and $\langle\varepsilon\rangle$ dropped to about 0.15 and $2 \times 10^{-4} \mathrm{~W} \mathrm{~kg}^{-1}$, respectively (Figs. $5 \mathrm{c}, \mathrm{d}$ ). The large wave steepness with high dissipation rate at the center of modulated groups suggests that the modulation instability increases steepness and triggers breaking events.

\section{b. Vertical profiles of TKE dissipation within a wave group}

So far we have examined the group phase-resolved nearsurface dissipation (Fig. 5d), and in the following we investigate the vertical structure of the TKE dissipation rate, especially during breaking and nonbreaking conditions. As illustrated in Fig. $5 \mathrm{~d}, \varepsilon$ varies with $\theta_{g}$, while having low and high dissipations approximately when $\left|\theta_{g}\right|<50^{\circ}$ and $\left|\theta_{g}\right|>$ $100^{\circ}$, respectively. To explore breaking and nonbreaking wave impacts on TKE dissipation, vertical profiles of TKE dissipation were averaged for $\left|\theta_{g}\right|<50^{\circ}$ and $\left|\theta_{g}\right|>100^{\circ}$ to represent dissipation under breaking $\varepsilon_{B}$ and nonbreaking $\varepsilon_{\mathrm{NB}}$ conditions, respectively. Figure 6 shows vertical profiles of $\varepsilon_{B}$ and $\varepsilon_{\mathrm{NB}}$ plotted as functions of $Z_{w} / H_{s}$ (Fig. 6a) and $Z / H_{s}$ (Fig. 6b), where $Z_{w}$ and $Z$ are the depths of the MRs from the 1-s-averaged wave surface and mean water line, respectively, and $H_{s}$ is the significant wave height computed as $H_{s}=4 \sigma^{2}$, where $\sigma$ is the variance of the wave elevation of the selected wave group segments (Table 1). Breaking wave dissipation $\varepsilon_{B}$ exhibits a two-layer structure (Fig. 6). Within the upper layer $\left(Z_{w} / H_{s}<0.5\right)$, the TKE dissipation rate is highly energetic with $\varepsilon_{B}$ as large as $3 \times 10^{-3} \mathrm{~W} \mathrm{~kg}^{-1}$, which is one order magnitude larger than $\varepsilon_{\mathrm{NB}}$. In the layer $\left(0.5<Z_{w} / H_{s}<1\right), \varepsilon_{B}$ decreases with depth more rapidly than $\varepsilon_{\mathrm{NB}}$. TKE dissipation rate $\varepsilon_{B}$ decreased to $O\left(10^{-5}\right) \mathrm{W} \mathrm{kg}^{-1}$ and was comparable with $\varepsilon_{\mathrm{NB}}$ at depths of $Z_{w} / H_{s}>1$. TKE dissipation vertical profiles in both reference frames $\left(Z_{w} / H_{s}\right.$ and $\left.Z / H_{s}\right)$ show similar results (Fig. 6). This may be due to the fact that 1-s-averaged depth $Z_{w}$ does not adequately represent wave-following depth.

A similar quasi-constant dissipation layer at shallower depth is also reported by Thomson et al. (2016) during breaking conditions. These observations suggest that the injected turbulence generated by breaking waves can significantly impact the vertical distribution of TKE dissipation in the near-surface layer for depths less than one wave height $\left(Z_{w} / H_{s}<1\right)$. In general, both vertical profiles of $\varepsilon_{B}$ and $\varepsilon_{\mathrm{NB}}$ can be approximated by a power 

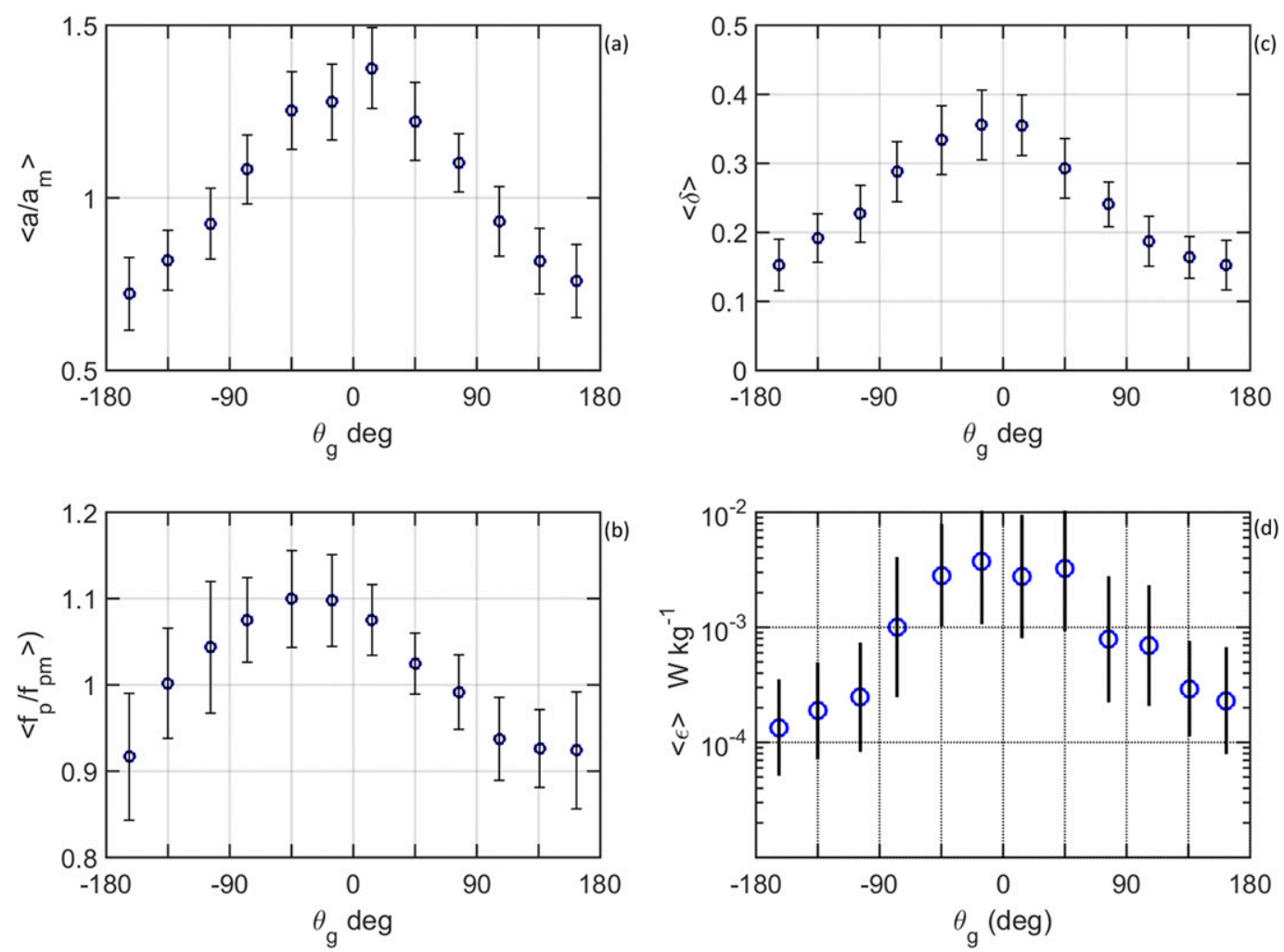

FIG. 5. Wave group phase dependence of bin-averaged (a) local wave amplitude $\left\langle a / a_{m}\right\rangle$, (b) wave frequency $\left\langle f_{p} / f_{p m}\right\rangle$, (c) steepness $\langle\delta\rangle$, and (d) TKE dissipation rate $\langle\varepsilon\rangle$. Data are from the test runs with the shear probe (MR140) at $0.16,0.19$, and $0.21 \mathrm{~m}$ below the mean water line. The $a_{m}$ and $f_{p m}$ are mean values of local amplitude and frequency, respectively, for each selected data segment (Table 1).

law relation with depth $Z_{w}^{-m}$, where $m$ is the exponent coefficient. For vertical profiles in the layer $\left(0.5<Z_{w} / H_{s}<1\right)$ during breaking waves, the dissipation profile of $\varepsilon_{B}$ decays rapidly with depth, where $m$ is about 7. Under nonbreaking wave conditions, $m$ is about 1.1, which is very similar to nonbreaking dissipation profiles reported by Lee et al. (2017). TKE dissipation in the open ocean tends to follow the similar power law, where $m$ varies from 1 to 2 (e.g., Sutherland and Melville 2015; Thomson et al. 2016). Open ocean observations represent phaseaveraged dissipation, and therefore $m$ tends to be smaller than in this study directly from the center of wave groups.

\section{Discussion}

\section{a. Comparison with open ocean observations}

The BF instability leads to modulation of the initially monochromatic wave train, and as a result, some waves grow at the expense of others (e.g., Benjamin and Feir 1967). Ultimately these steepening waves break and generate patches of energetic TKE dissipation rates on the surface (Babanin et al. 2010; Lee et al. 2017;
Schwendeman and Thomson 2017). Both local steepness and TKE dissipation rate peak near or at the center of the modulated wave group (Figs. 3 and 4). The observed periodicity of wave breaking associated closely with the modulated group is similar to observations from the open ocean described by Donelan et al. (1972), Holthuijsen and Herbers (1986), and Gemmrich and Farmer (2004).

Near-surface TKE dissipation rates in the ocean are on the order of $10^{-4}-10^{-2} \mathrm{~W} \mathrm{~kg}^{-1}$ for winds reaching $20 \mathrm{~m} \mathrm{~s}^{-1}$ and wave heights exceeding $7 \mathrm{~m}$ (Thomson et al. 2016). Similar dissipation rates were also found during moderate wind conditions (e.g., Gemmrich 2010; Schwendeman et al. 2014; Sutherland and Melville 2015). Gemmrich and Farmer (2004) measured TKE dissipation rates associated with individual breaking events at winds up to $14 \mathrm{~ms}^{-1}$. They showed that the background TKE dissipation at 1-m depth prior to the breaking event was about $5 \times 10^{-6} \mathrm{~W} \mathrm{~kg}^{-1}$ and that the dissipation at the same depth during breaking was as large as $8 \times 10^{-3} \mathrm{~W} \mathrm{~kg}^{-1}$. Furthermore, they reported that wave breaking occurred near the crest of the largest wave in the wave group. These ocean observations are 

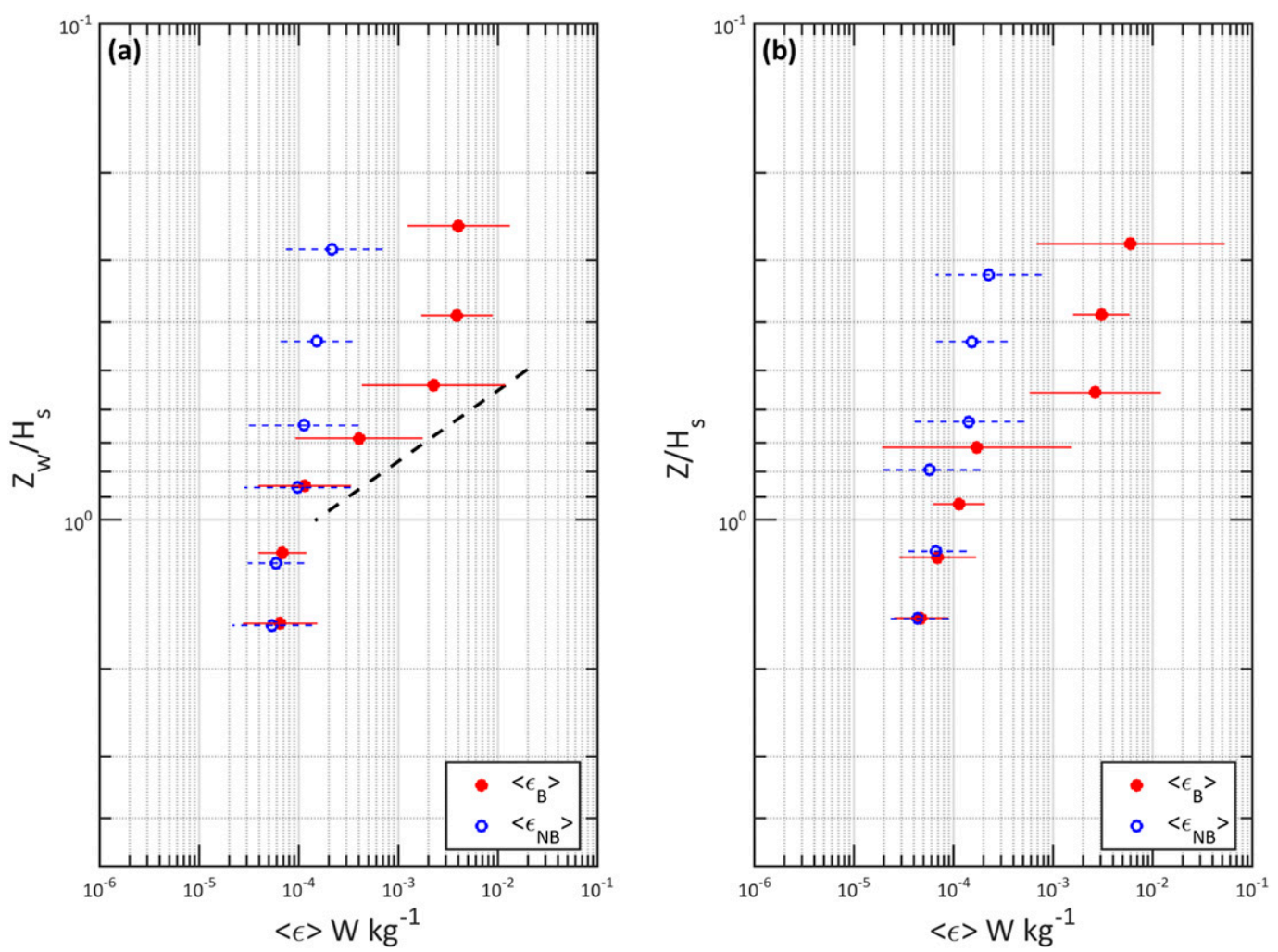

FIG. 6. Vertical profile of TKE dissipation in reference frame of (a) $Z_{w} / H_{s}$ and (b) $Z / H_{s}$. Solid red circles are for depth bin-averaged $\left\langle\varepsilon_{B}\right\rangle$, and blue open circles are for depth bin-averaged $\left\langle\varepsilon_{\mathrm{NB}}\right\rangle$. Horizontal solid lines represent $95 \%$ confidence intervals. The black dashed line in (a) represents $\varepsilon \sim\left(Z_{w} / H_{s}\right)^{-7}$.

consistent with the magnitude and pattern of dissipation enhancement during wave breakings in our tank experiments, even though the length and time scales of waves in the tank are much smaller than wave conditions in the open ocean. More recently, Callaghan et al. (2016) suggested that space- and time-averaged TKE dissipation rates in actively breaking waves are largely independent of length and time scales of breaking waves. They postulated that more energetic breaking waves increase air entrainment but not significantly increase the averaged turbulent intensity. The characterization and magnitude of TKE dissipation rates from this control experiment could be applicable to the open ocean conditions.

\section{b. Scaling of TKE dissipation rate of wave groups}

Based on dimensional considerations, the breaking wave TKE dissipation rate per unit mass $\varepsilon$ can be expressed as

$$
\varepsilon_{B}=A u_{t}^{3} / l_{t}
$$

where $u_{t}$ and $l_{t}$ are local turbulent velocity and length scales, respectively, and $A$ is a proportionality constant, which is an order one quantity (Tennekes and Lumley 1972). As discussed above the localized TKE dissipation rate within a breaking-wave patch varies a couple of orders of magnitude indicating that both $u_{t}$ and $l_{t}$ vary rapidly with time and space. In the absence of background mean flows, $u_{t}$ and $l_{t}$ are likely to be controlled by local wave properties such as orbital velocity and wavelength (Melville 1994; Babanin 2017). Here, $u_{t}$ is assumed to be proportional to wave orbital velocity $V_{\text {orb }}$ at the water surface and $l_{t}$ is assumed to be proportional to wavelength $L$. Using the linear dispersion relation, the turbulent velocity and length scales become $u_{t}=\alpha V_{\text {orb }}=\alpha(\delta C)$ and $l_{t}=\beta L=\beta\left(2 \pi C^{2} / g\right)$, where $C$ and $\delta$ are local wave phase speed and steepness, respectively, and $\alpha$ and $\beta$ are proportionality constants. By substituting $u_{t}$ and $l_{t}$ in terms of wave properties, the breaking wave TKE dissipation rate can be expressed as

$$
\varepsilon_{B}=\left(\frac{A \alpha^{3}}{\beta}\right) \delta^{3}\left(\frac{C^{3}}{L}\right)=B_{\mathrm{br}}\left(\frac{g C}{2 \pi}\right)
$$

where the nondimensional proportionality coefficient $B_{\mathrm{br}}$ is referred to as the breaking wave strength coefficient and is expressed as 


$$
B_{\mathrm{br}}=\left(\frac{A \alpha^{3}}{\beta}\right) \delta^{3}
$$

The TKE dissipation of per unit length of wave crest $\varepsilon_{L}$ is proportional to the phase speed of a breaking wave $C_{b}$, which is expressed as $\varepsilon_{L}=b \rho C_{b}^{5} / g$, where $\rho$ is the water density and $b$ is a nondimensional coefficient (Duncan 1981). The TKE dissipation rate per unit mass $\varepsilon$ is related to $\varepsilon_{L}$ as $\varepsilon=\varepsilon_{L} / \rho(D L / 2)$ (Melville 1994), where $L$ is wavelength and $D$ is the penetration depth of turbulent patch, which is typically proportional to the wavelength (e.g., Rapp and Melville 1990). By applying the linear dispersion relation, $D L \sim C^{4}$ and $C_{b} \sim C$, the TKE dissipation rate $\varepsilon$ derived from $\varepsilon_{L}$ can also be proportional to $C$ as shown in (5).

Thus, according to the dimensional consideration, the near-surface TKE dissipation rate is proportional to local phase speed with a breaking wave strength coefficient depending on local wave steepness. Here, the dependence of $B_{\mathrm{br}}$ on wave steepness [(6)] is examined for TKE dissipation rate measurements from the center of the wave groups (i.e., $\left|\theta_{g}\right|<50^{\circ}$ ) at depths of $0.16,0.19$, and $0.21 \mathrm{~m}$ (Table 1). Figure 7 shows a scatterplot of $B_{\text {br }}$ [i.e., $\left.\varepsilon_{B} /(g C / 2 \pi)\right]$ versus local steepness. The steepness bin-averaged $\left\langle B_{\mathrm{br}}\right\rangle$ increases rapidly with wave steepness and is approximately fitted by $\left\langle B_{\mathrm{br}}\right\rangle=0.015 \delta^{3}$ for $0.25 \leq \delta \leq 0.45$. This power-law dependence is consistent with the dimensional analysis in (6). Similar power-law dependence of breaking wave strength on steepness is shown in Romero et al. (2012).

\section{Conclusions}

In this study, we examine and quantify the spatial variability of TKE dissipation and its vertical profiles within a modulated wave group. The steep monochromatic waves generated by the wave maker in a 200-m-long wave tank, modulated rapidly into wave groups as they propagated downstream. Periods of the modulated wave groups were 4-10 times the initial periods of the monochromatic waves. The largest nearsurface TKE dissipation rates were found at or near the center of the modulated wave groups, where the wave steepness was largest. The observations suggest that the modulation instability steepens waves to their critical geometric limit at the onset of wave breaking. During wave-breaking events, the near-surface TKE dissipation can reach $O\left(10^{-2}\right) \mathrm{W} \mathrm{kg}^{-1}$, which is two to three orders of magnitude larger than before and after the wave breaking. The strongest TKE dissipation was limited to a near-surface layer within half of the significant wave height.

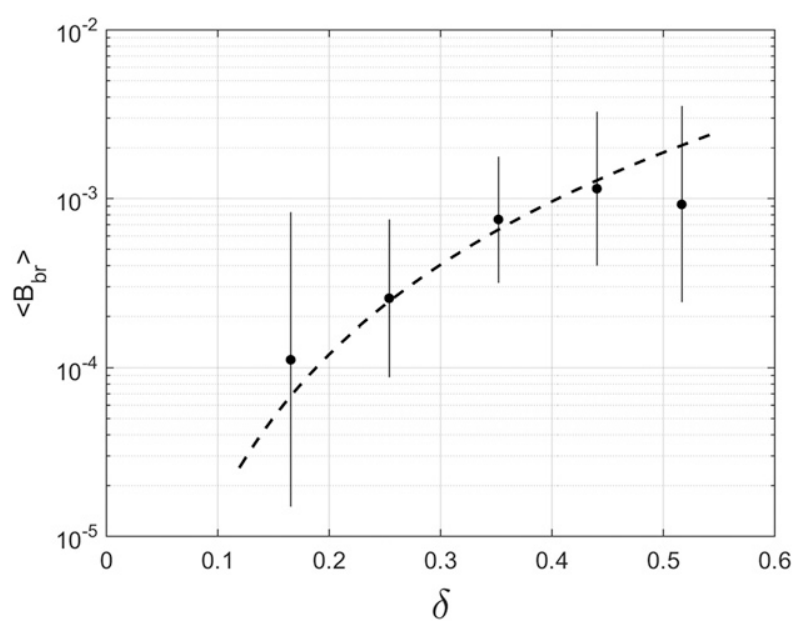

FIG. 7. The breaking wave strength coefficient $B_{\mathrm{br}}$ vs local wave steepness for near-surface TKE dissipation from the center of the wave group (i.e., $\left|\theta_{g}\right|<50^{\circ}$ ). Solid circles are steepness bin-averaged $\left\langle B_{\text {br }}\right\rangle$. Vertical solid lines represent $95 \%$ confidence intervals. The dashed line represents $B_{\mathrm{br}}=0.015 \delta^{3}$.

Vertical profiles of TKE dissipation rate for breaking $\varepsilon_{B}$ and nonbreaking $\varepsilon_{\mathrm{NB}}$ conditions were examined by averaging TKE energy dissipation estimates at or near the center of the wave group and away from the center of the wave group, respectively. The vertical profiles of $\varepsilon_{B}$ and $\varepsilon_{\mathrm{NB}}$ were significantly different in the nearsurface layer $\left(Z_{w} / H_{s}<0.5\right)$. The $\varepsilon_{B}$ in the near-surface depth layer is one order of magnitude larger than $\varepsilon_{\mathrm{NB}}$. At deeper depths, $\varepsilon_{B}$ decreased with depth more rapidly than $\varepsilon_{\mathrm{NB}}$ and became very comparable to $\varepsilon_{\mathrm{NB}}$ at depths larger than one wave height.

The TKE dissipation rate by modulated breaking waves can be parameterized by using local wave properties of phase speed and steepness, $\varepsilon_{B}=B_{\mathrm{br}}(g C / 2 \pi)$ and $\left\langle B_{\mathrm{br}}\right\rangle=0.015 \delta^{3}$. The characterization of near-surface TKE dissipation rate within the wave group and the parameterization using local wave properties can enhance our understanding and determination of energy dissipation of dominant wave breakings in open oceans.

Acknowledgments. This work was sponsored by the Bureau of Safety and Environmental Enforcement (BSEE), U.S. Department of the Interior Project 1059 titled "Characterizing wave-induced mixing energy in Ohmsett wave basin for dispersant effectiveness testing", under Contract E14PG00059. We thank Andrew Quaid, Ian Martens, and Justin Brodersen for their technical support during the experiment. Also thanks to Alan Guarino and Ohmsett staff for their help in the operation of the wave tank and the logistical support. We thank JPO Editor, Dr. Jerome Smith, and three anonymous reviewers for their constructive comments. 


\section{REFERENCES}

Asher, W., 2005: Data report for a wave characterization study at the Ohmsett wave basin. Minerals Management Service Rep. OSR-515, 179 pp., https:/www.bsee.gov/sites/bsee.gov/files/ osrr-oil-spill-response-research//515aa.pdf.

Babanin, A. V., 2017: Similarity theory for turbulence, induced by orbital motion of surface water waves. Procedia IUTAM, 20 99-102, https://doi.org/10.1016/j.piutam.2017.03.014.

_ - D. Chalikov, I. Young, and I. Savelyev, 2007: Predicting the breaking onset of surface water waves. Geophys. Res. Lett., 34, L07605, https://doi.org/10.1029/2006GL029135.

,,--- , and,- 2010 : Numerical and laboratory investigation of breaking of steep two-dimensional waves in deep water. J. Fluid Mech., 644, 433-463, https://doi.org/10.1017/ S002211200999245X.

Banner, M. L., and X. Tian, 1998: On the determination of the onset of wave breaking for modulating surface gravity water waves. J. Fluid Mech., 367, 107-137, https://doi.org/10.1017/ S0022112098001517.

- and W. L. Peirson, 2007: Wave breaking onset and strength for two-dimensional deep-water wave groups. J. Fluid Mech., 585, 93-115, https://doi.org/10.1017/ S0022112007006568

_ - A. V. Babanin, and I. R. Young, 2000: Breaking probability for dominant waves on the sea surface. J. Phys. Oceanogr., 30, 3145-3160, https://doi.org/10.1175/1520-0485(2000)030<3145: BPFDWO $>2.0 . \mathrm{CO} ; 2$

_- X. Xarthelemy, F. Fedele, M. Allis, A. Benetazzo, F. Dias, and W. L. Peirson, 2014: Linking reduced breaking crest speeds to unsteady nonlinear water wave group behavior. Phys. Rev. Lett., 112, 114502, https://doi.org/10.1103/ PhysRevLett.112.114502.

Benjamin, T. B., and J. E. Feir, 1967: The disintegration of wave trains on deep water. Part 1: Theory. J. Fluid Mech., 27, 417-430, https://doi.org/10.1017/S002211206700045X.

Bluteau, C. E., N. L. Jones, and G. N. Ivey, 2016: Estimating turbulent dissipation from microstructure shear measurements using maximum likelihood spectral fitting over the inertial and viscous subranges. J. Atmos. Oceanic Technol., 33, 713-722, https://doi.org/10.1175/JTECH-D-15-0218.1.

Callaghan, A. H., G. B. Deane, and M. D. Stokes, 2016: Laboratory air-entraining breaking waves: Imaging visible foam signatures to estimate energy dissipation. Geophys. Res. Lett., $\mathbf{4 3}$, 11320-11328, https://doi.org/10.1002/2016GL071226.

Donelan, M., M. S. Longuet-Higgins, and J. S. Turner, 1972: Periodicity in whitecaps. Nature, 239, 449-451, https://doi.org/ 10.1038/239449a0.

Drazen, D. A., W. K. Melville, and L. Lenain, 2008: Inertial scaling of dissipation in unsteady breaking waves. J. Fluid Mech., 611 307-332, https://doi.org/10.1017/S0022112008002826.

Duncan, J. H., 1981: An experimental investigation of breaking waves produced by a towed hydrofoil. Proc. Roy. Soc. London, 377A, 331-348, https://doi.org/10.1098/rspa.1981.0127.

— 1983: The breaking and non-breaking wave resistance of a two-dimensional hydrofoil. J. Fluid Mech., 126, 507-520, https://doi.org/10.1017/S0022112083000294.

Gemmrich, J., 2010: Strong turbulence in the wave crest region. J. Phys. Oceanogr., 40, 583-595, https://doi.org/10.1175/ 2009JPO4179.1.

-, and D. Farmer, 2004: Near-surface turbulence in the presence of breaking waves. J. Phys. Oceanogr., 34, 1067-1086, https://doi.org/10.1175/1520-0485(2004)034<1067:NTITPO > 2.0.CO;2.
Holthuijsen, L. H., and T. H. C. Herbers, 1986: Statistics of breaking waves observed as whitecaps in the open sea. J. Phys. Oceanogr., 16, 290-297, https://doi.org/10.1175/1520-0485(1986)016<0290: SOBWOA $>2.0 . \mathrm{CO} ; 2$.

Huang, Z. C., and H. H. Hwung, 2007: Local properties of wave modulation observed by wavelet analysis. 17th Int. Offshore and Polar Engineering Conf., Lisbon, Portugal, International Society of Offshore and Polar Engineers, ISOPE-I-07-176, https://www.onepetro.org/conference-paper/ISOPE-I-07-176.

Lee, J. H., J. P. Monty, J. Elsnab, A. Toffoli, A. V. Babanin, and A. Alberello, 2017: Estimation of kinetic energy dissipation from breaking waves in the wave crest region. J. Phys. Oceanogr., 47, 1145-1150, https://doi.org/10.1175/JPO-D-16-0273.1.

Lueck, R. G., F. Wolk, and H. Yamazaki, 2002: Oceanic velocity microstructure measurements in the 20th century. J. Oceanogr., 58, 153-174, https://doi.org/10.1023/A:1015837020019.

Melville, W. K., 1982: The instability and breaking of deep-water waves. J. Fluid Mech., 115, 165-185, https://doi.org/10.1017/ S0022112082000706.

1983: Wave modulation and breakdown. J. Fluid Mech.,128, 489-506, https://doi.org/10.1017/S0022112083000579.

1994: Energy dissipation in breaking waves. J. Phys. Oceanogr., 24, 2041-2049, https://doi.org/10.1175/1520-0485(1994) 024<2041:EDBBW>2.0.CO;2.

- , F. Veron, and C. J. White, 2002: The velocity field under breaking waves: Coherent structure and turbulence. J. Fluid Mech., 454, 203-233, https://doi.org/10.1017/ S0022112001007078.

Oakey, N. S., 1982: Determination of the rate of dissipation of turbulent energy from simultaneous temperature and velocity shear microstructure measurements. J. Phys. Oceanogr., 12, 256-271, https://doi.org/10.1175/1520-0485(1982)012<0256: DOTROD $>2.0 . \mathrm{CO} ; 2$

Perlin, M., W. Choi, and Z. Tian, 2013: Breaking waves in deep and intermediate waters. Annu. Rev. Fluid Mech., 45, 115-145, https://doi.org/10.1146/annurev-fluid-011212-140721.

Pizzo, N. E., and W. K. Melville, 2013: Vortex generation by deep-water breaking waves. J. Fluid Mech., 734, 198-218, https://doi.org/10.1017/jfm.2013.453.

Rapp, R., and W. K. Melville, 1990: Laboratory measurements of deep water breaking waves. Philos. Trans. Roy. Soc. London, 331A, 735-780, https://doi.org/10.1098/ rsta.1990.0098.

Romero, L., W. K. Melville, and J. M. Kleiss, 2012: Spectral energy dissipation due to surface wave breaking. J. Phys. Oceanogr., 42, 1421-1444, https://doi.org/10.1175/JPO-D-11-072.1.

Schwendeman, M. S., and J. Thomson, 2017: Sharp-crested breaking surface waves observed from a ship-based stereo video system. J. Phys. Oceanogr., 47, 775-792, https://doi.org/ 10.1175/JPO-D-16-0187.1.

- - - and J. Gemmrich, 2014: Wave breaking dissipation in a young wind sea. J. Phys. Oceanogr., 44, 104-127, https:// doi.org/10.1175/JPO-D-12-0237.1.

Soloviev, A., and R. Lukas, 2003: Observation of wave-enhanced turbulence in the near-surface layer of the ocean during TOGA COARE. Deep-Sea Res., 50, 371-395, https://doi.org/ 10.1016/S0967-0637(03)00004-9.

Sutherland, P., and W. K. Melville, 2015: Field measurements of surface and near-surface turbulence in the presence of breaking waves. J. Phys. Oceanogr., 45, 943-965, https:// doi.org/10.1175/JPO-D-14-0133.1.

Tennekes, H., and J. L. Lumley, 1972: A First Course in Turbulence. MIT Press, 320 pp. 
Thomson, J., M. S. Schwendeman, S. F. Zippel, S. Moghimi, J. Gemmrich, and W. E. Rogers, 2016: Wave-breaking turbulence in the ocean surface layer. J. Phys. Oceanogr., 46, 1857-1870, https://doi.org/10.1175/JPO-D-15-0130.1.

Tian, Z., M. Perlin, and W. Choi, 2010: Energy dissipation in twodimensional unsteady plunging breakers and an eddy viscosity model. J. Fluid Mech., 655, 217-257, https://doi.org/10.1017/ S0022112010000832.

Tulin, M. P., and T. Waseda, 1999: Laboratory observations of wave group evolution, including breaking effects. J. Fluid Mech., 378, 197-232, https://doi.org/10.1017/S0022112098003255.

Wang, D. W., and H. W. Wijesekera, 2017: Characterizing wave-induced mixing energy in OHMSETT wave basin for dispersant effectiveness testing. Bureau of Safety and Environmental Enforcement Tech. Rep. OSR-1059, 44 pp., https://www.bsee.gov/sites/bsee.gov/files/research-reports// 1059aa.pdf.

Zappa, C. J., W. R. McGillis, W. A. Raymond, J. B. Edson, E. J. Hintsa, H. J. Zemelink, J. W. H. Dacey, and D. T. Ho, 2007: Environmental turbulent mixing controls on air-water gas exchange in marine aquatic systems. Geophys. Res. Lett., 34, L10601, https://doi.org/10.1029/2006GL028790.

, M. L. Banner, R. P. Morison, and S. E. Brumer, 2016: On the variation of the effective breaking strength in oceanic sea states. J. Phys. Oceanogr., 46, 2049-2061, https://doi.org/10.1175/ JPO-D-15-0227.1. 